\title{
Orden de palabras, posición del objeto y estructura de la información en toba/qom (Guaycurú)
}

\section{Introducción}

La lengua toba toba/qom (familia Guaycurú) ha sido caracterizada de manera general como de orden flexible (Messineo 2003; Carpio y Censabella 2012). A otras lenguas de la familia se les ha atribuido también un orden flexible o libre: mocoví (Gualdieri 1998, Grondona 1998, Carrió 2015), pilagá (Vidal 2001), kaduveo (Sándalo 1997). No obstante, el orden de los constituyentes en la oración simple declarativa sigue un patrón relativamente estable de acuerdo con las relaciones gramaticales. Cuando la oración es transitiva, y el objeto es una frase nominal, el orden es AVO; cuando es intransitiva, es VS (Messineo 2003). A su vez, en las oraciones transitivas, un alineamiento jerárquico de persona $(1 / 2>3)$ tiene efecto sobre el orden de los constituyentes de manera que los objetos pronominales de primera y segunda persona preceden al verbo (orden OV) mientras que los de tercera se posponen (orden VO) (Messineo 2016).

Por otra parte, es sabido que las desviaciones del orden canónico en las lenguas (como las dislocaciones o las oraciones escindidas) se correlacionan con estructuras informacionales particulares (Payne 1992, 1993, Ward y Birner 2004, Guerrero y Belloro 2010). En toba, las estructuras de foco argumental (Lambrecht 1994) en el objeto de tercera persona muestran algún tipo de desviación del orden de palabras canónico y varios grados de integración sintáctica entre el elemento focalizado y el resto de la oración (Cúneo 2016a).

En el marco de los estudios de la interfaz entre sintaxis y pragmática, la posición del objeto puede concebirse como el locus en el que se negocian principios sintácticos y pragmáticos. En toba, cuando se trata de los participantes del acto de habla ( $1^{a} / 2^{a}$ persona) la posición preverbal del objeto es fija. Los objetos de tercera persona, en cambio, pueden ocupar una posición inesperada que, como mostraremos en este trabajo, está motivada pragmáticamente.

Con el objetivo general de examinar el interjuego entre el orden de palabras y la estructura de la información, nos proponemos, por un lado, revisar el orden de constituyentes en oraciones transitivas y detectar casos no canónicos

Paola Cúneo, Cristina Messineo, Consejo Nacional de Investigaciones Científicas y Técnicas y Universidad de Buenos Aires, Ciudad Autónoma de Buenos Aires, Argentina

Ә Open Access. (C) 2019 Paola Cúneo and Cristina Messineo, published by De Gruyter. (cc) BY-NC-ND This work is licensed under a Creative Commons Attribution-NonCommercial-NoDerivatives 4.0 International License. https://doi.org/10.1515/9783110605679-003 
de la posición del objeto y, por otro, explicar, desde la perspectiva de la estructura de la información, las motivaciones pragmáticas en las que el objeto presenta un ordenamiento no canónico.

Desde el punto de vista metodológico, el interés será identificar el orden básico de las oraciones transitivas a partir de un corpus textual heterogéneo que incluye géneros discursivos con distintas funciones comunicativas. Además de los textos, se incorporan herramientas de elicitación directa que permiten manipular contextos discursivos y obtener datos sobre estructuras informacionales específicas.

El capítulo se organiza de la siguiente manera. En lo que resta de la Introducción, se definen las categorías conceptuales utilizadas (1.1) y se expone la metodología de trabajo (1.2). La Sección 2 presenta un panorama general sobre la lengua toba (2.1), con especial atención a sus rasgos tipológicos más salientes y a la relación entre transitividad, jerarquía de persona y orden de los constituyentes (2.2). En la Sección 3 se expone la composición del corpus textual, se analizan los datos de acuerdo con la frecuencia y la distribución de la posición del objeto en oraciones transitivas y se identifican las construcciones en las que el objeto aparece en una posición no esperada. Con el aporte de nuevos datos obtenidos mediante la elicitación directa y los juicios gramaticales y de aceptabilidad de los hablantes, en la Sección 4 se analizan las motivaciones pragmáticas de las construcciones en las que el objeto aparece en posición preverbal. Por último, en la Sección 5 se exponen las conclusiones.

\subsection{Estructura de la información y orden no canónico}

Se asume aquí, de manera general, que la estructura de la información refiere al modo en el que los hablantes organizan las oraciones de acuerdo con sus necesidades comunicativas. Con el interés de estudiar cómo una lengua codifica en la gramática el estatus informativo que tienen los elementos en una oración, este trabajo se apoya en el enfoque de Lambrecht (1994), en el cual la organización gramatical de la información se define como estructura de foco, es decir, como la asociación convencional entre un significado focal y una forma oracional. Diferentes recursos prosódicos, morfológicos y sintácticos permiten indicar en las lenguas el alcance de la aserción en contraste con la presuposición en una emisión.

Además de la noción de foco de Lambrech (1944), como la parte de la aserción pragmática que difiere de la presuposición pragmática, este estudio considera también una taxonomía más específica de tipos de foco (cf. Dik et al. 1981, Drubig y Schaffar 2001) que permite explicar otras funciones comunicativas, más 
allá de la de completar un vacío de información. Serán de particular relevancia para nuestro análisis las categorías de foco restrictivo (cuya función es restringir un valor entre un conjunto dado previamente o presupuesto) y de foco de reemplazo (con la función de eliminar un ítem específico de la información asumida por el oyente y reemplazarlo por otro ítem asumido como correcto) (Dik et al. 1981). Como se observará más adelante, en toba cada tipo de foco se codifica mediante una construcción morfosintáctica diferente.

En cuanto a la interacción entre la estructura de foco y la sintaxis, los trabajos tipológicos reconocen que el orden de los constituyentes en las lenguas responde o bien a criterios sintácticos (como las relaciones gramaticales) o bien a principios pragmáticos (como los diferentes estatus informativos) (cf. Payne 1990, Van Valin 1999).

En esta misma línea de investigación, se asume en este trabajo que las desviaciones del orden canónico como, por ejemplo, una posición especial en la cláusula o la bipartición de la misma, se correlacionan con estructuras informacionales específicas (cf. Payne 1992, 1993, Ward y Birner 2004, Guerrero y Belloro 2010). Los fenómenos que se describen como preposicionamiento (preposing) y oración escindida (cleft) permitirán explicar los datos del toba.

$\mathrm{Si}$ bien se trata de un campo de estudio poco explorado en las lenguas indígenas chaqueñas (cf. Carpio 2009, Carpio 2011, Califa 2014, Califa y Belloro en prensa), los primeros avances sobre el tema en lo que respecta al posicionamiento del objeto mostraron que las estructuras de foco argumental en el objeto de tercera persona exhiben algún tipo de desviación del orden de palabras canónico y varios grados de integración sintáctica entre el elemento en foco y el resto de la oración (Cúneo 2016a). El presente capítulo avanza sobre esos resultados tanto desde el punto de vista teórico como metodológico, en tanto incorpora al análisis las categorías de preposicionamiento y oración escindida, y combina la observación del discurso natural (corpus de textos) con la manipulación de estímulos en tareas de elicitación directa.

\subsection{Metodología y corpus}

Los datos consignados en este trabajo pertenecen en su mayoría la variedad $\mathrm{da}$ pigemliek (noroeste de la provincia del Chaco) y fueron recogidos de primera mano por las autoras en sucesivos trabajos de campo. El corpus textual está conformado por distintos géneros discursivos (narrativas, consejos y rogativas) así como también por material narrativo obtenido de manera controlada mediante "la historia de la pera" (Chafe 1980), herramienta diseñada para el estudio del flujo de la información en narrativas (el hablante narra a partir de la 
observación de un video). Como complemento del material textual, ${ }^{1}$ se utilizó la elicitación directa con el objetivo de estudiar de manera específica los aspectos de la estructura de la información que son objeto de este trabajo. Para tal fin, se aplicó el Questionnaire for Information Structure (QUIS) (Skopeteas et al. 2006). Se trata de una herramienta específica para la investigación de la estructura de la información desde una perspectiva tipológica. El cuestionario posee tareas experimentales basadas en estímulos no verbales para la manipulación de las condiciones discursivas que pueden tener impacto en la estructura de la información de las emisiones. Esta herramienta evita la traducción de una lengua intermediaria, como el español. Para el presente trabajo, se seleccionaron algunas de las tareas centradas en la expectativa de uso de construcciones transitivas y ditransitivas, diseñadas especialmente para la elicitación de categorías pragmáticas de foco y tópico. En dichas tareas, se les solicitó a los hablantes la descripción de una situación o secuencia de situaciones, la narración de eventos, y los contextos de pregunta y respuesta, procurando combinar contextos verbales y no verbales. En algunos casos, se modificaron los escenarios para proveer situaciones culturalmente apropiadas y pertinentes al contexto chaqueño.

La metodología de análisis tuvo en cuenta los siguientes pasos. En primer lugar, se examinó el total de cláusulas transitivas que aparecen en el corpus textual y se identificaron los casos en los que el objeto aparece en una posición no canónica. Lo mismo se observó en la elicitación semidirigida de "la historia de la pera”. Un segundo paso fue aplicar el cuestionario (QUIS) y, una vez obtenidas las oraciones, se solicitaron juicios gramaticales a los hablantes y se aplicaron pruebas específicas, como la de la manipulación del orden del objeto y su posición relativa con respecto a la negación y otros elementos en la frase.

La comparación de los datos permitió arribar a tres tipos de construcciones en las que, de manera sistemática, el objeto aparece en posición preverbal.

\section{Lengua toba (Qom lPaqtaqa)}

\subsection{Generalidades}

La lengua toba (qom liaqtaqa) pertenece a la familia lingüística guaycurú, hablada en la región del Gran Chaco (Argentina, Bolivia y Paraguay). Su situación

\footnotetext{
1 La traducción que se provee en los textos difícilmente proporciona toda la información relevante y precisa para el análisis de fenómenos sensibles al contexto como, por ejemplo, las presuposiciones o la prominencia discursiva (cf. Matthewson 2004).
} 
dialectal es compleja y diversa; en Argentina, se identifican 5 áreas dialectales: dapigeml?ek (noroeste, Chaco), noPolgaGanaq (centro norte, Chaco), liañaGashek (centro sur, Chaco), takshek (sudeste, Chaco) y ñachilamol?ek (Formosa, Carpio 2012). La permanente movilidad dentro y fuera de la región chaqueña y la relocalización en comunidades urbanas es una de las causas del desplazamiento de la lengua y de la interrupción de la transmisión intergeneracional en contextos urbanos.

Desde el punto de vista tipológico, el toba es una lengua de marcación en el núcleo, con tendencia a la aglutinación y a la polisíntesis. Carece de adposiciones y de marcas de tiempo en el verbo. Cada verbo puede constituir por sí mismo una oración. Todas las formas verbales llevan obligatoriamente un índice de persona, independientemente de si los argumentos son expresados mediante frase nominal o pronombres independientes. El argumento sujeto también puede ser omitido $\mathrm{u}$ obviado (lengua pro-drop). Los índices distinguen participantes activos, inactivos y medios o activo-afectados. La $1^{\mathrm{a}}$ y la $2^{\mathrm{a}}$ persona presentan un alineamiento de intransitividad escindida de manera que $\mathrm{A}=\mathrm{Sa}$ y $\mathrm{O}=\mathrm{So}$, mientras que en la $3^{a}$ persona se observa un comportamiento diferente, más bien ligado a distintas escalas de transitividad (Messineo 2003).

Un rasgo característico de la lengua, que comparte con otras lenguas del Chaco, es el sistema de seis determinantes demostrativos que preceden al núcleo de la frase nominal e indican distancia y accesibilidad visual (próximo, distante y ausente) y posición (parado, sentado y acostado) del referente. Algunos de estos determinantes preceden también a las cláusulas relativas y completivas y cumplen funciones de relativizadores, complementantes y otros nexos entre predicados multiverbales (cf. Messineo y Porta 2009, Messineo en prensa).

La lengua carece de verbo copulativo, por lo cual la predicación nominal se obtiene mediante la yuxtaposción de dos nombres. Por último, el toba cuenta con una construcción existencial (wo?o) que, además de participar de diversos procesos morfológicos y sintácticos, funciona como foco presentacional en el discurso (Cúneo 2015).

\subsection{Transitividad, jerarquía de persona y orden de los constituyentes}

No existe una división tajante entre verbos transitivos e intransitivos. La transitividad depende tanto de la configuración fonológica de los índices 
pronominales, ${ }^{2}$ como de diversos mecanismos que permiten aumentar la valencia verbal. Los sufijos direccionales, locativos y comitativos funcionan como aplicativos, lo que permite el ascenso gramatical de un argumento oblicuo que se ubica siempre en la posición inmediatamente posverbal.

El toba posee también un alineamiento jerárquico que afecta la selección de los dos argumentos de un predicado transitivo indexados en el verbo (Messineo 2016). ${ }^{3}$ Los predicados transitivos permiten solo un índice de persona cuya selección depende de la posición relativa de los dos argumentos (A y O) sobre una jerarquía de persona en la que la $1^{\mathrm{a}}$ y la $2^{\mathrm{a}}$ comparten la posición más alta, mientras que la $3^{\mathrm{a}}$ ocupa la más baja. El argumento más alto en la jerarquía -independientemente de su rol gramatical- será el marcado:

\section{(1) zi-tawan ${ }^{4}$ 10(3A)-ayudar 'Él/ella me ayuda.' (1>3)}

Cuando A y O son tercera persona singular, la concordancia por defecto es con A. Este escenario sugiere que no es la jerarquía de persona la que opera sino una jerarquía de roles gramaticales en donde $A$ va a ser siempre el argumento marcado $(\mathrm{A}>0)$ :

\section{(2) i-tawan \\ 3A(30)-ayudar \\ 'Él/Ella lo/la ayuda.'}

La indexación de los argumentos centrales en el verbo permite la omisión de frases nominales en la cláusula, especialmente de A. No obstante,

2 Según la configuración fonológica de los prefijos de 3 a persona, el prefijo $i$ - $/ y$ - codifica participantes activos de verbos transitivos; $d$ - verbos de baja transitividad o intransitivos y $\emptyset$ - verbos intransitivos con participante inanimado o verbos locativos (Messineo 2003, 68).

3 Documentado por el momento solo en la variedad dapigeml?ek (Messineo 2016).

4 Abreviaturas: A (Agente); COL (colectivo); COM (comitativo); CON (conector discursivo); COORD (coordinante); DD (determinante demostrativo); DEM (demostrativo); DIM (diminutivo); DIR (direccional); F (femenino); IMP (impersonal); INTERR (interrogativo); LOC (locativo); MASC (género masculino); NEG (negativo); NX (nexo); O (Objeto); PL (plural); POS (marcador de poseedor); PRO (pronombre personal); PROG (aspecto progresivo); PROM (marcador de prominencia discursiva); 1 (primera persona); 2 (segunda persona); 3 (tercera persona); 4 (persona indefinida); \# (pausa). 
también $\mathrm{O}$ puede omitirse y su identidad establecerse mediante el rastreo de la referencia ${ }^{5}$ :

\section{(3) $\boldsymbol{n}$-tageñi (a-da l-yale) \\ 3A(30)-peinar F-DD 3POS-hijo(F)}

'La peina (a su hija).'

Respecto del orden de los constituyentes, los estudios sobre la lengua (Messineo 2003; Carpio 2007; Carpio y Censabella 2012, entre otros) identifican un orden básico no marcado AVO para las oraciones transitivas y VS para las intransitivas. No obstante, respecto de las oraciones intransitivas, los trabajos mencionados observan cierta variación en la posición del sujeto intransitivo (VS y SV). ${ }^{6}$

Por otra parte, en lo que respecta a la posición de un $\mathrm{O}$ pronominal de primera y segunda persona, se observa un comportamiento sintáctico también regido por la jerarquía de persona $(1 / 2>3)$, de manera que los pronombres independientes de primera/segunda persona preceden siempre al verbo (orden OV), mientras que el de tercera se pospone (VO) (Messineo 2016) ${ }^{7}$ :

\section{(4) ayem n-tageñi \\ 1PRO 3A-peinar \\ 'Me peina.' (1>3) \\ (5) Pam i-tawan \\ 2PRO 3A-ayudar \\ 'Te ayuda.' (2>3)}

5 En una construcción ditransitiva, no obstante, el Tema debe explicitarse mediante una frase nominal y el Recipiente puede omitirse e inferirse del contexto:

6 Un estudio reciente sobre el orden no canónico de cláusulas intransitivas SV en mocoví y en toba muestra que la animacidad, el estatuto informativo y la accesibilidad cognitiva son las principales motivaciones semánticas y pragmáticas para la posición de S preverbal (Califa y Belloro en prensa).

7 Pruebas con la posición del adverbio y de la negación confirmarían la colocación de los O pronominales $1^{\mathrm{a}}$ y $2^{\mathrm{a}}$ vs. $3^{\mathrm{a}}$, dentro y fuera del alcance de la frase verbal (Messineo 2016). 


\section{(6) i-tawan damaze \\ 3A-(30)-ayudar 3PRO(MASC) \\ 'Él/Ella lo ayuda.' (3A>30)}

Cuando dos argumentos nominales de $3^{\mathrm{a}}$ persona singular co-ocurren, el objeto (con rol semántico de Paciente, Tema o Recipiente) ocupa la posición fija posverbal (7a), de manera que la alteración del orden de los argumentos afecta la interpretación de la cláusula (7b):
(7) a. so nataGala? i-tawan so nsoqolek
DD jefe 3A(30)-ayudar DD joven
'El jefe ayudó al joven.'
b. so nsoqolek i-tawan so nataGala?
DD joven 3A(30)-ayudar DD jefe
'El joven ayudó al jefe.'

Por último, un objeto aplicado -con rol semántico de locativo (8)-(9), instrumental (10), o comitativo (11) - se comporta de manera idéntica que en los casos consignados en (7):

(8) kishigem-ek a-da Pepaq

3.subir-LOC F-DD árbol

'(El/ella) sube al árbol.'

(9) a-da Palo i-waGan-gi na l-qayk da yale F-DD mujer 3-pegar-LOC DD 3POS-cabeza DD hombre

'La mujer le pega en la cabeza al hombre.'

(10) a-da Palo i-waGan-gi da Repaq

F-DD mujer 3-pegar-LOC DD árbol

'Le pega con el palo.'
(11) $\tilde{n}$-awan-e? so Virgilio 1-encontrar-COM DD Virgilio
'Me encontré con Virgilio.'

\section{Posición del argumento objeto en textos orales}

El corpus de textos está compuesto por dos relatos extensos, veinte consejos y veinte rogativas (publicado en parte en Messineo 2014). Se incluye también el 
recuento de la historia de la pera (Chafe 1980) elicitado para la lengua por las autoras.

Como es común en el estilo narrativo, los relatos se caracterizan por una alta frecuencia de terceras personas (tanto frases nominales como pronombres independientes y demostrativos), así como también por el uso del existencial woPo con función presentacional (Cúneo 2015).

En cambio, en los consejos y las rogativas, géneros de estilo persuasivo, predominan los pronombres independientes de $2^{\mathrm{a}}$ persona (en los consejos) y $1^{\mathrm{a}}$ persona (en las rogativas), y los verbos transitivos, la mayoría de ellos con función apelativa (Messineo 2009, 2014, Cúneo y Messineo 2017).

La Tabla 1 muestra las características generales del corpus seleccionado.

Tabla 1: Corpus de textos en toba/qom.

\begin{tabular}{lrl}
\hline Género & Total de Cláusulas & Estilo \\
\hline 2 narrativas & 300 & Narrativo \\
1 “HISTORIA DE LA PERA” & 84 & Narrativo \\
20 consejos & 256 & Persuasivo \\
20 rogativas & 86 & Persuasivo \\
TOTAL & 726 & \\
\hline
\end{tabular}

El conteo de los datos tomó en cuenta todas las cláusulas transitivas en donde el objeto es una frase nominal, un pronombre independiente o un demostrativo. Se dejaron de lado, no obstante, los objetos de naturaleza clausal. Se incluyeron objetos típicos con rol semántico de Paciente, Tema y Recipiente, así como también los objetos aplicados con rol Locativo, Instrumental y Comitativo. En casos en los cuales en una misma cláusula co-ocurren dos objetos $($ Tema $=$ frase nominal $\mathrm{y}$ Recipiente $=$ pronombre independiente de $1^{\mathrm{a}}$ o $2^{\mathrm{a}}$ ), se optó por contar sólo el Tema.

El corpus que se describe arriba exhibe un total de 280 cláusulas transitivas, en las cuales el objeto se codifica como frase nominal, como demostrativo o bien como pronombre personal independiente (de $1^{a}, 2^{a}$ y $3^{a}$ persona). La distribución de cláusulas según la codificación de la persona en el argumento $\mathrm{O}$ se presenta en la Tabla 2.

Tabla 2: Cláusulas transitivas según la codificación de la persona en el argumento 0.

\begin{tabular}{lrr}
\hline Clásulas transitivas & 0 = FN, DEM Y PRON 3 & 0 = PRON 1 \\
\hline 280 & 252 & y 2 \\
\hline
\end{tabular}


En cuanto a la posición del objeto, la frecuencia relativa de cada tipo de orden (VO/OV) se presenta en la Tabla 3.

Tabla 3: Cláusulas transitivas según la posición de 0 .

\begin{tabular}{lr}
\hline Vo & OV \\
\hline 238 & 42 \\
$(85 \%)$ & $(15 \%)$ \\
\hline
\end{tabular}

Como era de esperar, el orden más frecuente es VO, que representa el 85\% del total de las cláusulas transitivas. Ahora bien, si analizamos el total de cláusulas con orden VO, observamos en la Tabla 4 que ninguno de los objetos corresponde a un pronombre independiente de $1^{\mathrm{a}} \mathrm{o}$ de $2^{\mathrm{a}}$ persona (Participantes del Acto de Habla). Todos los argumentos posverbales de objeto refieren a una $3^{a}$ persona (no Participantes del Acto de Habla), codificada preferentemente como frase nominal.

Tabla 4: Cláusulas transitivas con orden Vo.

\begin{tabular}{lr}
\hline & VO \\
\hline O = PAH & O = noPAH \\
\hline 0 & 238 \\
$(0 \%)$ & $(100 \%)$ \\
\hline
\end{tabular}

Por último, la Tabla 5 muestra que, de los objetos preverbales del corpus, el $33 \%$ corresponde a objetos de $3^{a}$ persona (la mayoría de ellos codificados como frases nominales), mientras que el $67 \%$ corresponde al total de casos de $1^{\mathrm{a}}$ y $2^{\mathrm{a}}$ persona.

Tabla 5: Cláusulas transitivas con orden OV.

\begin{tabular}{lr}
\hline \multicolumn{2}{c}{ OV } \\
\hline O = PAH & O = nOPAH \\
\hline 28 & 14 \\
$(67 \%)$ & $(33 \%)$ \\
\hline
\end{tabular}


En síntesis, el análisis de los textos muestra que, en distintos géneros discursivos, la posición del objeto es predominantemente fija: preverbal, cuando se trata de pronombres PAH y posverbal en el caso de pronombres de tercera persona, frases nominales y demostrativos.

La baja frecuencia relativa del orden OV con objetos nominales de $3^{\mathrm{a}}$ persona sugiere que, si bien este tipo de ordenamiento no es agramatical, está pragmáticamente marcado, como se verá en el análisis. Las siguientes secciones se centran en las motivaciones pragmáticas de tres tipos de construcciones en las que el argumento objeto de 3a persona ocurre en posición preverbal.

\section{Posición preverbal del objeto y motivaciones pragmáticas}

Cuando una construcción ubica un constituyente que es canónicamente posverbal en una posición preverbal se denomina "preposicionamiento" (preposing) (Ward y Birner 2004). Desde el punto de vista formal, el constituyente prepuesto se caracteriza porque ocurre dentro de los límites clausales; es decir, preserva sus roles sintáctico y semántico como complemento del verbo (Lambrecht 2001a).

Ward y Birner (2004) proponen dos tipos mayores de preposicionamiento: la topicalización y el preposicionamiento focal. Si bien ambos tipos se distinguen por su función informativa (así como también por sus propiedades prosódicas), en ambos casos existe una relación con el discurso previo. El argumento en la posición prepuesta involucra típicamente un referente relativamente familiar o "dado", y este referente puede estar relacionado con el discurso precedente de diversas maneras (mediante relaciones de tipo-subtipo, entidad-atributo, parte-todo, identidad, etc.). En el caso del preposicionamiento focal, la entidad prepuesta constituye un miembro alternativo de un conjunto, que es dado en el discurso.

A partir de la elicitación directa en complemento con el análisis de los ejemplos documentados en los textos identificamos, a la par que la estructura canónica (12a), tres construcciones en las que el objeto ocupa una posición preverbal: las construcciones con verbo impersonal qa-/qo- (12b), las construcciones con el adverbio ndoteek (12c), y las escisiones de objeto (12d).

(12) a. a-da Palo y-asot-ek a-ñi peloota F-DD mujer 3-patear-LOC F-DD pelota 'La mujer pateó la pelota.' 
b. $a-\tilde{n} i$ peloota qa-y-asot-ek

F-DD pelota IMP-3-patear-LOC

'La pelota patearon.'

c. ndoteek a-ñi peloota y-asot-ek a-da Palo solo F-DD pelota 3-patear-LOC F-DD mujer

'Sólo la pelota pateó la mujer.'

d. peloota \# zi y-asot-ek a-da Palo

pelota DD 3-patear-LOC F-DD mujer

'Una pelota (fue) lo que pateó la mujer.'

Si bien todas las oraciones son sintácticamente diferentes, son equivalentes desde el punto de vista semántico y son aceptables en contextos discursivos particulares. Como veremos a continuación, están en correlación con motivaciones pragmáticas específicas.

En primer lugar, observamos que (12a) presenta el orden AVO y se asocia con una estructura de foco de predicado (Lambrecht 1994). Por otra parte, los ejemplos en (12b) y (12c) pueden ser analizados como construcciones de preposicionamiento (Ward y Birner 2004). Estas involucran un constituyente referencial objeto en una posición preverbal, no canónica. No obstante, estas construcciones difieren en los recursos morfosintácticos involucrados y, fundamentalmente, en su función pragmática. Finalmente, en el ejemplo en (12d) se observa una construcción escindida o cleft (Lambrecht 2001b).

En los siguientes apartados se detallan las propiedades morfosintácticas, prosódicas y funcionales (informacionales) de los tres tipos de construcciones, que constituyen estructuras focales alternativas al tipo de foco de predicado codificado en (12a).

\subsection{Preposicionamiento de objeto en construcciones con qa-/qo- 'impersonal'}

Existe en toba un tipo de construcción impersonal (Messineo 2003, 73) que se forma mediante el morfema $q a$-/qo- que se añade mayormente a verbos transitivos flexionados en $3^{a}$ persona singular, e indica que se desconoce el agente o que su identidad carece de relevancia. ${ }^{8}$

8 Este prefijo es presentado también como indicador de una construcción pasiva no promocional en dos variedades de toba (Carpio y González 2017), mediante la cual se destopicaliza el agente de la cláusula activa, pero sin promoción del argumento P. 
Si bien esta construcción presenta un orden canónico VO, en algunos casos ocurre junto a frases nominales prepuestas con función sintáctica de objeto. El siguiente ejemplo ilustra este fenómeno; obsérvese que el verbo concuerda con el objeto prepuesto mediante el infijo -?- que indica plural de objeto:

(13) nache ñi nsoq qataq a-ñi qa?añ-ole qa-y-apaGage?n OV CON DD joven COORD F-DD joven.F-DIM IMP-3-enseñar.PLO

'Entonces al joven y a la jovencita se les enseña.'

Desde el punto de vista funcional, la construcción de preposicionamiento de objeto junto al prefijo de impersonal da cuenta de un alto grado de topicalidad del referente del constituyente objeto. De acuerdo con Givón (1983), la topicalidad se asocia con la continuidad con el discurso previo y con la persistencia con el discurso subsiguiente. Además, en términos de Prince (1992), un referente topical debe ser dado para el oyente (hearer-old), es decir, identificable para el oyente al momento de la emisión, así como también dado en el discurso (discourse-old), es decir, evocado de alguna manera en el discurso previo o en el contexto extra-lingüístico. El referente entonces posee cierto grado de saliencia o continuidad en el discurso, como se observa en el siguiente ejemplo:

(14) a. Contexto previo: 'Entonces comieron los qom. Comieron la carne de la vaca. Esperaban el tren para irse. Y se fueron los qom. [...] Entonces se llevaron a los qom, en ese momento los qom se fueron a Salta en siete vagones de carga. Cuando llegaron a Salta entonces hicieron trabajar a los qom cortando la madera, cortando el rolizo, cortando postes y leñas. También todos los chicos de trece años, quince años trabajaban también cortando la madera (porque) cuando habían llegado todavía no estaba lista la caña. En Salta (la gente) no entendía la forma (el clima del lugar).'

b. Nache ne?ena maiche l-ma? yi?iyi Salta CON DEM propio 3POS-lugar DEM Salta

na qom qa-y-amaq-tege-lek

DD toba IMP-3-ordena-PROG-LOC

da chek a-na qo-y-itega coca

NX 3.comer F-DD IMP-3-decir coca

'Entonces la gente del propio lugar de Salta a los qom obligó a masticar eso que llaman coca.’ 
El constituyente prepuesto (na qom 'los tobas, los qom') en (14b) refiere a una entidad previamente introducida en el contexto discursivo y reiteradamente mencionada en cláusulas anteriores. No obstante, dado que se han introducido otros referentes discursivos en las intervenciones inmediatamente previas, se trata de una entidad que no se encuentra máximamente "activa” (Chafe 1987) y que, por lo tanto, se recupera mediante la construcción prepuesta.

En otros casos, la fuente de identificabilidad del referente se encuentra en el contexto social y en el conocimiento compartido por la comunidad de habla. Este es el caso de "la jovencita y el jovencito" en el nqataGak ('consejo'), dado que son los destinatarios culturalmente apropiados de este tipo de discurso. La jovencita y el jovencito son los referentes del constituyente prepuesto en varios consejos de nuestro corpus (véase (13) arriba, y los ejemplos en (15) y (16)). En (16), nótese que la identificabilidad del referente se manifiesta también mediante el uso de recursos gramaticales específicos, como el demostrativo o el sufijo - $m$ que se adjunta a los determinantes. ${ }^{9}$

(15) a-so qa?añ-ole nataqa?en qa-y-apaGagen-ek da l-ataGak F-DD joven.F-DIM también IMP-3-enseñar-LOC DD 3POS-costumbre da weta-?a ze l-ma? DD estar-DIR DD 3POS-casa

'A la jovencita también se le enseña la costumbre de estar en su casa.'

(16) nache sePeso nsoq-ole-k qataq a-so qa?añ-ole CON DEM joven.M-DIM-M con F-DD joven.F-DIM qataq imawek ze-m ziPizi l-yal-aq-pi so-m n-ta?a CON todo DD-PROM DEM 3POS-hijo-PL-COL DD-PROM 3POS-padre qa-y-amaGa-lek da saishet.da chek sePeso shigiyak IMP-3-ordenar-LOC NX NEG 3.comer DEM animal 'Entonces a ese jovencito y a esa jovencita y también a todos los hijos de esa familiase les ordena que no deben comer ese animal.'

Desde el punto de vista sintáctico, la marca de impersonal ( $q a$-) evita que un objeto en posición preverbal se interprete como sujeto, dado que, como vimos en la Sección 2.1, cuando dos terceras personas co-ocurren, las relaciones gramaticales están codificadas en el orden de los constituyentes. De esta manera, la posición

\footnotetext{
9 Según Messineo (2003, 171-180), el marcador pragmático - $m$ puede funcionar para otorgar prominencia a ciertos tramos o elementos del discurso o bien como marcador de referencialidad discursiva (Givón 1979, Du Bois 1980), en relación con la continuidad e importancia que posee un ítem a lo largo del discurso.
} 
preverbal del objeto con un verbo de $3^{a}$ persona sin marca de impersonal afecta la interpretación de la cláusula. Contrástense los ejemplos en (17a) y (17b):

(17) a. Bruno qa-y-amaGañi OV

Bruno IMP-3-empujar

'A Bruno lo empujaron.'

b. Bruno y-amaGañi $\mathrm{AV}(\mathrm{O})$

Bruno 3-empujar

'Bruno empujó (a alguien).'

Con respecto al estatus sintáctico del constituyente prepuesto, observamos que este forma parte integral de la cláusula. A diferencia de la construcción escindida, que se analiza en la Sección 4.3, las construcciones prepuestas se incluyen en la misma unidad entonacional (no hay presencia de pausa) y no puede intervenir ningún elemento entre la frase nominal inicial y el resto de la cláusula (18b), criterios que permiten identificar estas construcciones como casos de preposicionamiento (cf. Lambrecht 2001a, Ward y Birner 2004).

(18) a. a-ñi peloota qa-y-asot-ek

F-DD pelota IMP-3-patear-LOC

'la pelota patearon'

b. $a$-ñi peloota ${ }^{*} a-\tilde{n} i$ qa-y-asot-ek

F-DD pelota F-DD IMP-3-patear-LOC

'la pelota *la patearon'

En síntesis, las construcciones de preposicionamiento con verbos que llevan la marca de impersonal constituyen un recurso para lograr comunicar la topicalidad de un referente objeto (en consonancia con la omisión del agente) y evitar un objeto topical en la posición canónica de foco de predicado.

Las construcciones impersonales con objeto prepuesto admiten objetos con varios roles semánticos, como Recipiente (19) o Tema (20).

(19) Cris qataq Paola qa-y-apaGagen-ek na qom l-?aqtaqa

Cris COORD Paola IMP-3-enseñar-LOC DD toba 3POS-idioma

'A Cris y a Paola les enseñan el idioma toba.'

(20) na qom l-Paqtaqa qa-y-apaGagen-ek añi Cris qataq Paola

DD toba 3POs-idioma IMP-3-enseñar-LOC F-DD Cris COORD Paola

'El idioma toba les enseñan a Cris y a Paola.' 
Además de las frases nominales, un demostrativo puede también ocupar la posición prepuesta, en consonancia con la tendencia que presentan las lenguas en cuanto a la codificación de argumentos: las formas más "reducidas" codifican participantes más activos mientras que las frases nominales plenas típicamente introducen participantes nuevos. El siguiente ejemplo corresponde a un fragmento de un relato sobre la peligrosidad del chancho salvaje, entidad previamente introducida en el contexto discursivo y cognitivamente activa:

(21) a. Contexto: Entonces al otro día lo buscamos a ese chancho. Lo encontraron donde estaban. Llevaban lanzas, pero no es como la escopeta. Entonces muchos chanchos fueron heridos, sí, con la lanza, pero lo lastima nomás. Entonces casi casi me mata (el chacho), y subí a un árbol. Entonces parecía que yo estaba como muerto allá arriba. Entonces me caí. Y ese (chancho) es el que casi te mató. Porque muchos chanchos ya están muertos.

b. anaPana qa-i-lotege

DEM IMP-3-vigilar

'Esos (chanchos) están muy vigilados;

a-na qa-i-kemaqchet

F-DD IMP-3-lastimar

a esos (chanchos) hirieron.'

Cuando se trata de un referente mencionado en el discurso previo pero no activo, es posible que, además de expresarse mediante un demostrativo (anaPana) con función sintáctica de objeto en la posición prepuesta, necesite también codificarse mediante una frase nominal plena (ana lanza 'la lanza') en una posición dislocada a la derecha con la función de antitópico (Lambrecht 2001a), lo que confirma el valor topical del referente (la lanza).

nache qalaGaze ana?ana qa-y-ache-tak \# a-na lanza
CON COORD DEM IMP-3-llevar-PROGR F-DD lanza
'pero a esa llevaban, a la lanza'

\subsection{Preposicionamiento de objeto en construcciones con ndoteek 'solo'}

Otro tipo de construcciones prepuestas que también involucran un constituyente referencial con función sintáctica de objeto en una posición preverbal no canónica es aquella que ocurre con el adverbio ndoteek ‘solo'. Al igual que en 
las construcciones analizadas en la Sección 4.1, el constituyente prepuesto ocurre dentro de los límites de la cláusula y funciona por lo tanto como un complemento del verbo. Sin embargo, ambos tipos de construcciones difieren en su función pragmática dado que, en este caso, el constituyente prepuesto se corresponde con el dominio del foco (Focus preposing, Ward y Birner 2004) y no del tópico.

En los siguientes ejemplos se observa que la forma adverbial ndoteek 'solo' (cf. Cúneo 2016b) funciona como un adverbio restrictivo o "exclusivo" (exclusive) (cf. Koenig 1991) cuya función es la de excluir o restringir un elemento (en foco) entre un conjunto de potenciales alternativas que constituyen información dada o presupuesta (Dik et al. 1981, Renans et al. 2016). El adverbio ndoteek interviene en construcciones con el orden OV cuando el objeto coincide con el dominio del foco argumental de tipo restrictivo ${ }^{10}$ :

(23) a-so Graciela ndoteek a-so amap n-dogo $\mathrm{AOV}^{11}$

F-DD Graciela solo F-DD algarroba 3-traer

‘Graciela solo ALGARROBA buscó’ (Interpretación: no buscó otro tipo de frutos)

(24) so Rufino ndoteek a-ñi l-wa n-qopita AOV

DD Rufino solo F-DD 3POS-esposa 3-amar

'Rufino ama solo A SU ESPOSA' (Interpretación: no ama a nadie más)

(25) ndoteek aso n-tongishi chek aso Ana OVA

solo F-DD 4POS-tortilla 3.comer F-DD Ana

'Solo TORTILLA comió Ana' (Interpretación: no comió otra cosa)

(26) ndoteek a-zi banana de-shejnaGana aso Cacho OVA

solo F-DD banana 3-comprar F-DD Cacho

'Solo BANANA compró Cacho' (Interpretación: no compró otra cosa)

(27) ndoteek a-zi nere i-taGayaGaa-tak da Juan OVA

solo F-DD libro 3-leer-PROG DD Juan

'Solo LA BIBLIA está leyendo Juan’ (Interpretación: no lee otros libros)

10 El foco se señala en versalitas en los ejemplos.

11 Los factores que condicionan la posición de A en los ejemplos exceden el alcance de este trabajo. 
De acuerdo con su función pragmática de expresión de foco restrictivo, estas construcciones poseen características prosódicas que las distinguen de las preposiciones de tópico presentadas en la Sección 4.1. Dado que toda la oración, excepto el objeto prepuesto, codifica información pragmáticamente presupuesta, el constituyente prepuesto es el único punto de prominencia prosódica en la oración; por lo tanto, el objeto lleva el acento nuclear y el resto de la cláusula es desacentuado.

$\mathrm{Al}$ igual que en las construcciones con qa- (Sección 4.1), los objetos prepuestos con ndoteek admiten varios grados de animacidad y distintos roles semánticos, Tema (28), Recipiente (29) y Locativo (30):

(28) ndoteek so Pepaq y-anem a-da ?alo solo DD árbol 3-dar F-DD mujer

'Sólo EL PALO le dio a la mujer.'

(29) ndoteek ada Palo y-anem da Pepaq

solo F-DD mujer 3-dar DD árbol

'Solo A LA MUJER le dio el palo'

(30) a-da Palo ndoteek na lqayk i-waGan-gi da yale

F-DD mujer solo DD 3POS-cabeza 3-pegar-LOC DD hombre

so Pepaq

DD árbol

'La mujer solo EN LA CABEZA le pegó al hombre con el palo'

\subsection{Construcciones escindidas}

Finalmente, observamos un conjunto amplio de casos que presentan una estructura diferente a las construcciones analizadas en las Secciones 4.1 y 4.2, que analizamos como construcciones escindidas (clefts). De manera general, una construcción escindida involucra una organización sintáctica que se utiliza para distinguir un constituyente que es discursivamente prominente del resto de la oración. Se divide, por lo tanto, en dos partes, una de ellas en foco y otra en segundo plano (cf. Lambrecht 2001b, Hartmann y Veenstra 2013).

Los siguientes ejemplos ilustran una estructura biclausal con las siguientes particularidades: está conformada por un elemento nominal (sin determinante) en posición inicial y una cláusula finita (equiparable a una relativa) encabezada por un determinante correferencial con el nominal inicial, con el cual concuerda en género, número, posición o distancia. 
(31) yale \# da i-waGan-gi na l-qayk

hombre DD 3-pegar-LOC DD 3POS-cabeza

'A UN/EL HOMBre (fue) al que le pegó en la cabeza.'

(32) Paola \# a-ñi yoqta s-awatton

Paola F-DD realmente 1-conocer

na l-ayi-pi saishet

DD 3POS-otro-COL NEG

'A PAOLA (es) a la que realmente conozco. A los otros, no.'

(33) a. y-achet-tak da nki?aGala?

3-llevar-PROG DD mesa

'¿Está llevando una mesa?'

b. saishet \# so?onaGaki \# a-da y-achet-tak

NEG silla F-DD 3-llevar-PROG

'No, uNA SILla (es) lo que está llevando'

El carácter biclausal de estas construcciones se confirma mediante la prueba con la negación, dado que es posible negar la primera cláusula de manera independiente mediante la negación clausal saishet da, como en (34a) y (35a), en contraste con los correlatos uniclausales en los ejemplos en (34b) y (35b) que ocurren con la negación verbal sa:

(34) a. saishet.da Virgilio \# so ñ-awan-e?

NEG Virgilio DD 1-ver-COM

'No (fue) A VIRGILIO a quien vi.'

b. sa ñ-awan-e? ka Virgilio (=1 cláusula)

NEG 1-ver-COM DD Virgilio

'No vi a Virgilio.'

(35) a. sa.ishet da l-qayk \# na i-wanGan-gi

NEG DD 3POS-cabeza DD 3-pegar-LOC

'No (fue) EN LA CABEZA donde (le) pegó.

b. sa i-waGan-gi na l-qayk (=1 cláusula)

NEG 3-pegar-LOC DD 3Pos-cabeza

'No (le) pegó en la cabeza.'

Además, las construcciones escindidas en toba admiten la inversión sintáctica de los constituyentes. Dado que la lengua no posee verbo copulativo, ambos constituyentes se encuentran yuxtapuestos. 
(36) a. pyoq lta?adayk \# so s-elap-a

perro grande DD 1-ver-LOC

'UN PERRO GRANDE (fue) lo que vi.'

b. so s-elar-a \# pyoq ltaradayk

DD 1-ver-LOC perro grande

'Lo que vi (fue) un perro grande.,'

La estructura biclausal expresa, no obstante, una única proposición semántica, que puede ser también expresada en una cláusula simple sin modificar sus condiciones de verdad:

(37) a. Pepaq \# so y-anem (a-da ?alo)

árbol DD 3-dar F-DD mujer

'Un PALO (fue) lo que le dio (la mujer).'

b. a-da ?alo y-anem so Pepaq

F-DD mujer 3-dar DD árbol

'La mujer le dio un palo.'

En cuanto a su función pragmática, las oraciones escindidas que documentamos en toba ocurren en contextos de contraste. Según Drubig y Schaffar (2001, 1079), hay contraste cuando existe un conjunto de alternativas disponible especificado por contexto, ya sea verbal o no verbal, y se selecciona un miembro de este conjunto. En particular, cuando el hablante elimina un ítem específico de la información asumida por el oyente y lo reemplaza por otro ítem que asume como correcto, Dik et al. (1981) llaman a esta función "foco de reemplazo" (replacing focus). En los siguientes ejemplos, las respuestas constituyen construcciones escindidas que tienen la función de corregir las expectativas creadas en la pregunta y reemplazarlas por el referente correcto.

$\begin{array}{llll}\text { saishet.da } & \text { kishigem-ek } & a-d a & \text { mapik } \\ \text { NEG } & \text { 3.subir-LOC } & \text { F-DD } & \text { algarrobo }\end{array}$

'No sube al algarrobo (Prosopis alba).'

12 La identificación y la caracterización de las oraciones escindidas en toba teniendo en cuenta una tipología funcional amplia (clefts, pseudo-clefts, invertidas, presentacionales; cf. Lambrecht 2001b, Hartmann y Veenstra 2013) excede el alcance del presente trabajo. Por lo mismo, no consignamos aquí el tipo de estructura focal que se corresponde con construcciones como las de (37b). 


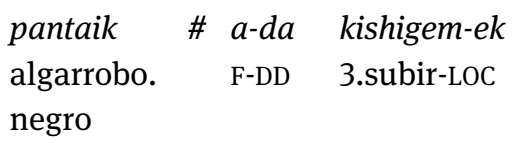

Al ALGARRoBo NEGRo (Prosopis nigra) (es) al que sube.'

(39) saishet.da i-waGan-gi na l-qayk

NEG 3-pegar-LOC DD 3POS-cabeza

'No le pegó en la cabeza.

l-waq \# na i-waGan-gi

3POS-mano DD 3-pegar-LOC

EN LA MANO (fue) donde le pegó.’

En consonancia con la descripción de Dik et al. (1981, 63) del foco de reemplazo, en los ejemplos en toba pueden distinguirse varias etapas: la negación (sa ishet) y el rechazo del referente incorrecto - mapik 'algarrobo' en (38) y lqayk 'su cabeza' en (39)-, y la sustitución por el correcto - pantaik 'algarrobo negro' y lwaq 'su mano'-.

En síntesis, esta estrategia permite focalizar el referente del objeto semántico, indicando que la oración no debe interpretarse como foco de predicado (tópico-comentario). Como se observa en los ejemplos anteriores, las construcciones escindidas permiten focalizar diferentes roles semánticos, tales como Recipiente en (31) y (32), Tema en (33) y (37) y Locativo en (35) y (38), algunos de los cuales constituyen objetos aplicados.

Finalmente, la lengua permite formular preguntas escindidas ${ }^{13}$ :

(40) mapik \# a-da kishigem-ek

algarrobo F-DD 3.subir-LOC

¿¿(Es) al algarrobo al que sube?’

(41) l-qayk \# na i-waGan-gi

3POS-cabeza DD 3-pegar-LOC

‘¿Fue) en la cabeza donde le pegó?'

(42) Pepaq \# da i-waGan-gi

árbol DD 3-pegar-LOC

¿¿Es con el palo que le pega?’

13 La función de las preguntas con estructura escindida, así como su relación con las preguntas "neutrales" (no escindidas) necesita más estudio. 


\section{Conclusiones}

El trabajo examinó el orden de palabras en oraciones transitivas en la lengua toba sobre la base de un corpus textual amplio y variado en estilos discursivos.

El análisis del corpus confirmó que la lengua presenta un orden rígido para los objetos pronominales de $1^{\mathrm{a}}$ y $2^{\mathrm{a}}$ persona (OV), mientras que para las $3^{\mathrm{a}}$ personas (pronombres independientes, demostrativos y frases nominales) se observa una alta frecuencia de VO. No obstante, en algunos casos, el objeto ocurre en una posición preverbal. Esta posición se correlaciona con y está motivada por varias funciones pragmáticas.

A partir de la elicitación directa, los resultados de la investigación mostraron que las desviaciones del orden canónico del objeto se correlacionan con tres tipos de construcciones que en términos informacionales se corresponden con fenómenos de preposicionamiento (preposing) y oración escindida (cleft) y que codifican tópico, foco restrictivo y foco de reemplazo. Estas construcciones se distinguieron en base a sus propiedades sintácticas, prosódicas e informacionales.

Los criterios específicos que permitieron distinguir los tipos de construcciones documentados fueron los siguientes. a) el estatus sintáctico de los constituyentes y los límites clausales; b) los recursos morfosintácticos propios de cada construcción; c) la ocurrencia de un determinante demostrativo correferencial con el constituyente objeto; d) la prosodia; y e) la función informativa.

En base a estos criterios, observamos dos tipos de construcciones prepuestas. Por un lado, aquellas que ocurren junto a verbos que llevan la marca de impersonal $q a$ - y que constituyen un recurso para comunicar la topicalidad de un referente objeto. Por otro, las construcciones que ocurren con el adverbio ndoteek 'solo', en las cuales, a diferencia de las anteriores, el constituyente prepuesto contiene el foco de la emisión con una interpretación restrictiva. Desde el punto de vista formal, en ambos tipos de construcciones el constituyente prepuesto ocurre dentro de los límites de la cláusula y funciona como un complemento del verbo. En términos informacionales, en ambos casos el constituyente prepuesto denota una entidad discursiva de alguna manera relacionada con el discurso precedente. Se distinguen, además, por su función informativa específica: tópico, en el caso de las construcciones impersonales, y foco restrictivo, en el caso aquellas con ndoteek 'solo'.

A diferencia de los casos de preposicionamiento, los datos permitieron observar un tipo de estructura escindida - biclausal- en la que el constituyente escindido tiene la función de foco de reemplazo. En comparación con las anteriores, estas construcciones presentan un menor grado de 
integración sintáctica, tal como lo confirman la pausa marcada entre las cláusulas y la prueba de que cada cláusula puede ser negada de manera independiente.

La Tabla 6 sintetiza las principales características de los tipos de construcciones documentadas.

Tabla 6: Tipos de construcciones y funciones pragmáticas.

\begin{tabular}{llllll}
\hline & $\begin{array}{l}\text { Límites } \\
\text { clausales }\end{array}$ & $\begin{array}{l}\text { Elemento } \\
\text { pronominal } \\
\text { correferencial }\end{array}$ & $\begin{array}{l}\text { Otros recursos } \\
\text { morfosintácticos }\end{array}$ & $\begin{array}{l}\text { Prominencia } \\
\text { prosódica }\end{array}$ & Función \\
\hline $\begin{array}{l}\text { Preposicionamiento } \\
\text { OV }\end{array}$ & intraclausal & no & qa-/qo- & no & tópico \\
\hline $\begin{array}{l}\text { Preposicionamiento } \\
\text { OV }\end{array}$ & intraclausal & no & ndoteek & sí & $\begin{array}{l}\text { foco } \\
\text { restrictivo }\end{array}$ \\
\hline $\begin{array}{l}\text { Construcciones } \\
\text { escindidas }\end{array}$ & biclausal & sí & $\begin{array}{l}\text { determinante } \\
\text { correferencial en } \\
\text { la segunda } \\
\text { cláusula }\end{array}$ & sí & $\begin{array}{l}\text { foco de } \\
\text { reemplazo }\end{array}$ \\
& & & & & r \\
\hline
\end{tabular}

Para concluir, podemos afirmar que en toba los objetos más "marcados" sintácticamente -es decir, que ocurren en una posición inicial inesperada- se asocian o bien con un tópico o bien con algún tipo de foco de contraste (restrictivo y de reemplazo) ligado al discurso previo.

Con el interés en las funciones y usos comunicativos que hacen los hablantes, el trabajo intentó mostrar que las construcciones que presentan un orden no canónico del objeto son sistemáticas y se usan de manera consistente para organizar la información de acuerdo con necesidades comunicativas específicas.

\section{Agradecimientos}

Esta investigación se realizó gracias al apoyo económico del CONICET (Consejo Nacional de Investigaciones Científicas y Técnicas de Argentina) y de la Universidad de Buenos Aires (Proyecto UBACyT 2016-2019 20020160100106BA). Agradecemos a los evaluadores anónimos y a la editora del presente volumen por sus valiosos comentarios. 


\section{Referencias}

Califa, Martín. 2014. La Estructura Argumental Preferida en mocoví (guaycurú): Proyecciones teórico-metodológicas. Signo y Seña / Revista del Instituto de Lingüística 25. 9-34.

Califa, Martín y Valeria Belloro. En prensa. Motivaciones discursivas para la posición de sujeto intransitivo en mocoví y toba (guaycurúes, Argentina). Lingüística 36 (1).

Carpio, Belén. 2007. Sistemas de alineación en toba (familia guaycurú, Argentina). Tesis de Maestría. Universidad de Sonora.

Carpio, Belén. 2009. Accesibilidad referencial en toba (flia. guaycurú, Argentina). Revista Estudios en Ciencias Humanas. Estudios y monografías de los postgrados de la Facultad de Humanidades (UNNE).1-11.

Carpio, Belén. 2011. Morfosintaxis y usos pragmáticos de los demostrativos en toba ñachilamole’k (Formosa, Argentina). En Ana Fernández Garay y Antonio Díaz-Fernández (eds.). Investigaciones sobre lenguas indígenas sudamericanas, 19-40. Santa Rosa, La Pampa: Universidad Nacional de La Pampa.

Carpio, Belén. 2012. Fonología y morfosintaxis de la lengua hablada por grupos tobas en el oeste de Formosa (Argentina). München: LINCOM.

Carpio, Belén y Marisa Censabella. 2012. Clauses as noun modifiers in Toba (Guaycuruan). En Bernard Comrie y Zarina Estrada-Fernández (eds.). Relative Clauses in the Languages of the Americas. A typological overview, 173-189. Amsterdam/Philadelphia: John Benjamins.

Carrió, Cintia. 2015. Construcciones causativas y anticausativas en mocoví. LIAMES 15(1). 69-89.

Chafe, Wallace (ed.). 1980. The Pear Stories: Cognitive, Cultural, and Linguistic Aspects of Narrative Production. Norwood, New Jersey: Ablex.

Chafe, Wallace. 1987. Cognitive Constraints on Information Flow. En Russell Tomlin (ed.). Coherence in Discourse and Grammar, 21-51. Amsterdam: John Benjamins.

Cúneo, Paola. 2015. De la existencia al foco. Polifuncionalidad de las construcciones existenciales con woßo en toba (guaycurú). En Zarina Estrada-Fernández, Ana Fernández Garay y Albert Álvarez González (coord.). Estudios de lenguas amerindias 3. Escenarios de diversidad lingüística, 285-311. Hermosillo: Unison.

Cúneo, Paola. 2016a. Expresión de foco en toba (guaycurú). Una aproximación al interjuego entre sintaxis y estructura de la información. Cuadernos de la ALFAL, Revista de la Asociación de Lingüística y Filología de América Latina 9. 64-79.

Cúneo, Paola. 2016b. No solo ... sino también ... en toba (guaycurú). Foco expansivo y formas de habla. Ponencia presentada en el Segundo Congreso Internacional. Los Pueblos Indígenas de América Latina. Santa Rosa: Universidad Nacional de La Pampa, 20-24 de septiembre.

Cúneo, Paola y Cristina Messineo. 2017. NatamnaGakpi 'Rogativas.' El arte de pedir y suplicar entre los qom. En Andrés Dapuez y Florencia Tola (comp.). El Arte de Pedir: Antropología de dueños y suplicantes, 25-57. Villa María: EDUVIM (Editorial Universitaria de Villa María).

Dik, Simon, Maria Hoffman, Jan de Jong, Sie Ing Djang, Harry Stroomer y Lourens de Vries. 1981. On the typology of focus phenomena. En Teun Hoekstra (ed.). Perspectives on Functional Grammar, 41-74. Dordrecht: Foris.

Drubig, Hans Bernhard y Wolfram Schaffar. 2001. Focus constructions. En Martin Haspelmath (ed.). Language Typology and Language Universals: An International Handbook, vol. II, 1079-1104. Berlin/New York: Mouton de Gruyter. 
Du Bois, John. 1980. Beyong Definiteness: The Trace of Identity in Discourse. En Wallace Chafe (ed.). The Pear Stories: Cognitive, Cultural and Linguistic Aspects ff Narrative Production, 203-174. Norwood: Ablex.

Givón, Talmy. 1979. On Understanding Grammar. New York: Academic Press.

Givón, Talmy. 1983. Topic continuity in discourse. A quantitative cross-language study. Amsterdam: John Benjamins.

Gonzalez, Raúl y Belén Carpio. 2017. Causativización perifrástica en toba del este y del oeste de la provincia de Formosa (Argentina). Íkala 22(3). 439-454.

Grondona, Verónica. 1998. A grammar of Mocoví. Tesis de doctorado. University of Pittsburgh.

Guadieri, Beatriz. 1998. Mocoví (Guaycurú). Fonologia y morfossintaxe. Tesis de doctorado. Universidade Estadual de Campinas.

Guerrero, Lilián y Valeria Belloro. 2010. On word order and information structure in Yaqui. En José Camacho, Rodrigo Gutiérrez Bravo y Liliana Sánchez (eds.). Information Structure in Indigenous Languages of the Americas, 115-137. Berlin/Boston: Mouton de Gruyter.

Hartmann, Katharina y Tonjes Veenstra (eds.). 2013. Cleft Structures. Amsterdam/ Philadelphia: John Benjamins.

König, Ekkehard. 1991. The Meaning of Focus Particles. A Comparative Perspective. London/ New York: Routledge.

Lambrecht, Knud. 1994. Information Structure and Sentence Form: Topic, Focus, and the Mental Representations of Discourse Referents. Cambridge: Cambridge University Press.

Lambrecht, Knud. 2001a. Dislocation. En Martin Haspelmath (ed.). Language Typology and Language Universals, 1050-1078. Berlin/New York: Mouton de Gruyter.

Lambrecht, Knud. 2001b. A framework for the analysis of cleft constructions. Linguistics 39(3). 463-516.

Matthewson, Lisa. 2004. On the Methodology of Semantic Fieldwork. International Journal of American Linguistics 70(4). 369-415.

Messineo, Cristina. 2003. Lengua toba (guaycurú). Aspectos gramaticales y discursivos. München: LINCOM.

Messineo, Cristina. 2009. Estructura retórica, recursos lingüísticos y función social del nqataGak (consejo toba). Revista Signos 42(70). 197-218.

Messineo, Cristina. 2014. Arte Verbal Qom: consejos, rogativas y relatos tobas. Textos bilingües anotados (Con textos de Mauricio Maidana). (Ethnographica). Buenos Aires: Rumbo Sur.

Messineo, Cristina. 2016. Indexación y orden: huellas de alineamiento jerárquico en toba (Guaycurú). Ponencia. XV Congreso de la Sociedad Argentina de Lingüística, Bahía Blanca: Universidad Nacional del Sur.

Messineo, Cristina. En prensa. Syntactic Complexity and Grammaticalization in Toba Language. En Zarina Estrada-Fernández, Albert Álvarez González y Claudine Chamoreau (eds.). Diverse scenarios of syntactic complexity: inter and intra typological diversity. Amsterdam/Philadelphia: John Benjamins.

Messineo, Cristina y Andrés Porta. 2009. Cláusulas relativas en toba (guaycurú). International Journal of American Linguistics 75(1). 49-68.

Payne, Doris. 1990. The Pragmatics of Word Order: Typological Dimensions of Verb Initial Languages. Berlin/New York: Mouton de Gruyter.

Payne, Doris (ed.). 1992. Pragmatics of Word Order Flexibility. Amsterdam/Philadelphia: John Benjamins. 
Payne, Doris. 1993. On the function of word order in Yagua narrative. International Journal of American Linguistics 59(1). 1-15.

Prince, Ellen. 1992. Subjects, definiteness, and information-status. En William Mann y Sandra Thompson (eds.). Discourse Description. Diverse Linguistic Analyses of a Fund-raising Text, 295-326. Amsterdam/Philadelphia: John Benjamins.

Renans, Agata, Malte Zimmermann y Makcus Greif. 2016. Questionnaire on Focus Semantics. Working Papers of the SFB 632. Interdisciplinary Studies on Information Structure (ISIS) 15.

Sándalo, Filomena. 1997. A Grammar of Kadiwéu. With Special Reference to the Polysynthesis Parameter. Cambridge: MIT.

Skopetas, Stavros, Ines Fiedler, Sam Hellmuth, Anne Schwarz, Ruben Stoel, Gisbert Fanselow, Caroline Féry y Manfred Krifka. 2006. Questionnaire on Information Structure (QUIS). Reference Manual. Interdisciplinary Studies on Information Structure 4.

Van Valin, Robert. 1999. A Typology of the Interaction of Focus Structure and Syntax. En Ekatarina Raxilina and Jakov Testelec (eds.). Typology and the Theory of Language: From Description to Explanation. Moscow: Languages of Russian Culture.

Vidal, Alejandra. 2001. Pilagá Grammar (Guaycuruan Family, Argentina). Tesis de doctorado. University of Oregon.

Ward, Gregory y Betty Birner. 2004. Information Structure and Non-Canonical Syntax. En Laurence Horn y Gregory Ward (eds.). The Handbook of Pragmatics, 153-174. Oxford: Blackwell. 